\title{
Response Analysis on Electrical Pulses under Severe Nuclear Accident Temperature Conditions Using an Abnormal Signal Simulation Analysis Module
}

\author{
Kil-Mo Koo, ${ }^{1}$ Jin-Ho Song, ${ }^{1}$ Sang-Baik Kim, ${ }^{1}$ Kwang-Il Ahn, ${ }^{2}$ Won-Pil Baek, ${ }^{3}$ \\ Kil-Nam Oh, ${ }^{4}$ and Gyu-Tae Kim ${ }^{5}$ \\ ${ }^{1}$ Severe Accident and PHWR Safety Research Division, Korea Atomic Energy Research Institute, 150 Dukjin-dong, Yusong-gu, \\ Daejeon 305-353, Republic of Korea \\ ${ }^{2}$ Integrated Safety Assessment Division, KAERI, 150 Dukjin-dong, Yuseong-gu, Daejeon 305-353, Republic of Korea \\ ${ }^{3}$ Nuclear Safety Research Headquarter, KAERI, 150 Dukjin-dong, Yuseong-gu, Daejeon 305-353, Republic of Korea \\ ${ }^{4}$ Department of Optical Communications Engineering, Gwangju University, 52 Hyuodeok-ro, Nam-gu, \\ Gwangju 503-703, Republic of Korea \\ ${ }^{5}$ Department of Electrical Engineering, Korea University, 5ka, Anam-dong, Sungbuk-ku, Seoul 136-701, Republic of Korea
}

Correspondence should be addressed to Kil-Mo Koo, kmkoo@kaeri.re.kr

Received 7 December 2011; Revised 19 March 2012; Accepted 19 March 2012

Academic Editor: Gilberto Espinosa-Paredes

Copyright (c) 2012 Kil-Mo Koo et al. This is an open access article distributed under the Creative Commons Attribution License, which permits unrestricted use, distribution, and reproduction in any medium, provided the original work is properly cited.

\begin{abstract}
Unlike design basis accidents, some inherent uncertainties of the reliability of instrumentations are expected while subjected to harsh environments (e.g., high temperature and pressure, high humidity, and high radioactivity) occurring in severe nuclear accident conditions. Even under such conditions, an electrical signal should be within its expected range so that some mitigating actions can be taken based on the signal in the control room. For example, an industrial process control standard requires that the normal signal level for pressure, flow, and resistance temperature detector sensors be in the range of $4 \sim 20 \mathrm{~mA}$ for most instruments. Whereas, in the case that an abnormal signal is expected from an instrument, such a signal should be refined through a signal validation process so that the refined signal could be available in the control room. For some abnormal signals expected under severe accident conditions, to date, diagnostics and response analysis have been evaluated with an equivalent circuit model of real instruments, which is regarded as the best method. The main objective of this paper is to introduce a program designed to implement a diagnostic and response analysis for equivalent circuit modeling. The program links signal analysis tool code to abnormal signal simulation engine code not only as a one body order system, but also as a part of functions of a PC-based ASSA (abnormal signal simulation analysis) module developed to obtain a varying range of the $R-C$ circuit elements in high temperature conditions. As a result, a special function for abnormal pulse signal patterns can be obtained through the program, which in turn makes it possible to analyze the abnormal output pulse signals through a response characteristic of a $4 \sim 20 \mathrm{~mA}$ circuit model and a range of the elements changing with temperature under an accident condition.
\end{abstract}

\section{Introduction}

To diagnose a severe nuclear accident, it is essential to determine a plant's status and to continuously monitor a plant's responses, expected from the actions taken by operators to mitigate a given accident [1]. In addition, a correct interpretation of a plant's conditions during such an accident is of significant importance for a successful accident management [2]. According to SECY 89-012, the United State Nuclear Regulatory Commission (U.S. NRC) requires that in a reasonable way instrumentation is identified as one of the key elements in utility accident management plants [3]. Moreover, a review of a plant's accident management capabilities is regarded as a crucial element in achieving a regulatory closure for severe accident issues. During accidents, information and data from a plant's instruments are essential in assessing the status and response of that plant. There are some inherent uncertainties of the 
reliability of instrumentations expected while subjected to harsh environments occurring in severe nuclear accident conditions. Even for a severe accident condition, however, there are a few ways to obtain the relevant information and data. According to $[4,5]$, for example, circuit simulation analysis and diagnosis methods are available to assess these instruments in detail even when they provide apparently abnormal readings. Then, some abnormal signal diagnostics and analysis could be made through modeling of an important circuit that is composed of passive elements $(R-$ $L-C)$, an input multifunction generator, and an output pulse measurement system. These simulations would be useful for investigating what the signal and circuit response characteristics would be, similar to the relevant symptoms that can be obtained under severe environmental conditions as a quality analysis method [5].

In this paper, a newly enhanced simulation module has been designed through the realization of a one body order system [6] via one program that links an abnormal signal simulation engine program (PSpice and Multi-SIM code) to a signal analysis tool program (LabVIEW code), which includes signal analysis functions from an important circuit model applicable under the conditions [7]. In that case, the aforementioned abnormal signal simulation engine is used as a one order command which means a nonstop processing command to import the simulation data from an output of the engine code to an input of the main analysis tool program. The design of the above simulation module can be broken up into three main steps [8]. The first step is a section of a comparative assessment of the instruments between the instrument specifications or EQ (environment qualification) requirement parameters and the environment parameters that can be obtained through a severe accident simulation code such as MAAP4 whose mean circumstance data is obtained through a simulation of various accident scenarios [9]. From the results, if its comparative result is not satisfied, the second step is a section of the design of a circuit simulation to apply an abnormal signal simulation processing technique with an equivalent circuit, the third step is a section of a signal analysis of functions that have an additional 5 substeps. Consequently, three main functions exist and are characterized as an abnormal signal processing, an accident management, and an additional guide that are displayed on the initial screen of a simulation system ASSA module. Using the ASSA module, we could obtain the response characteristics from the output voltage levels of a pulse parameter according to a change of the resistance for each of $R_{2}$, and $R_{3}$, and the response characteristic from the output voltage level and time constants of the pulse parameter according to a change of the capacitance for each of $C_{1}$, and $C_{2}$.

As a result, when we can analyze the cause from an equivalent circuit, which consists of an $R-C$ distribution parameter, from a response characteristic for a $4 \sim 20 \mathrm{~mA}$ instrument during high temperature conditions of the severe accident conditions (SACs), it is also possible to detect and diagnose the abnormal signal patterns of a malfunctioning instrument. In addition, the pulse parameter estimation is operable to estimate a pulse parameter for a signal based on an analysis of the detected pulses and information pertaining to there being abnormal pulses in the signal as a quantitative analysis method.

When this simulation module is set up in a laboratory, we can obtain a variety of abnormal signals simulated under degraded conditions like higher temperature conditions of the SACs. A large variety of built-in models and a simulator adds flexibility to a simulation and includes the effect of harsh environments by using a special function to identify the components exceeding a manufactures' limits from a circuit modeling including the $R-C$ passive elements.

We present the design steps with the diagnosis methods and the circuit simulation using a specific signal processing in Section 2, followed by a detailed description of work carried out during the past five years of our diagnostics and analysis work in Sections 3-5. Some concluding remarks are presented in Section 6 and a reference of papers resulting from the project is included.

\section{Design Steps of Diagnosis Methods and the Circuit Simulation with Specific Signal Processing}

\begin{abstract}
2.1. Design Steps of Diagnosis Methods for the Malfunctioning Instruments. When an instrument which is providing information and data for severe accident management is apparently malfunctioning, a series of steps can be taken which include an indirect diagnosis of the instrument and a direct method of determining the value of the parameter. These methods are identified by five steps which are called operational aids. They are combinations of diagnostic actions and other means of measurement. These combinations can be applied when instrument readouts are suspect or faulty. The five types of aids are a diverse indication, parameter inference, a portable instrument, circuit diagnosis, and a portable circuit readout [4] (Figure 1). In this case, a circuit diagnosis from among the diagnostic evaluations belongs to action items for a malfunctioning instrument where a circuit diagnosis using a simulation tool code is possible.
\end{abstract}

2.2. Design Steps of the Circuit Simulator. Figure 2 shows an initial screen consisting of functions for the ASSA module. It has three main functions which have not only an abnormal signal processing function, but also a severe accident management function based on EPRI TR-103412 and EPRI TR102371 technical reports including a comparative assessment procedure for instruments $[4,5]$, and an additional guide on the initial screen of an ASSA module.

Figure 3 shows a flowchart of the overall design steps for the simulator as an ASSA module system. The comparative assessment procedure for the instruments has the main information and data including the information needs for the instrument specifications, position information according to five areas in the containment building, and the circumstance parameters from the accident scenarios using the MAAP4 code. Figure 4(a) shows the three EQ conditions which include a normal condition, an abnormal condition, and an out of range condition. Figure 4(b) shows five phases of 


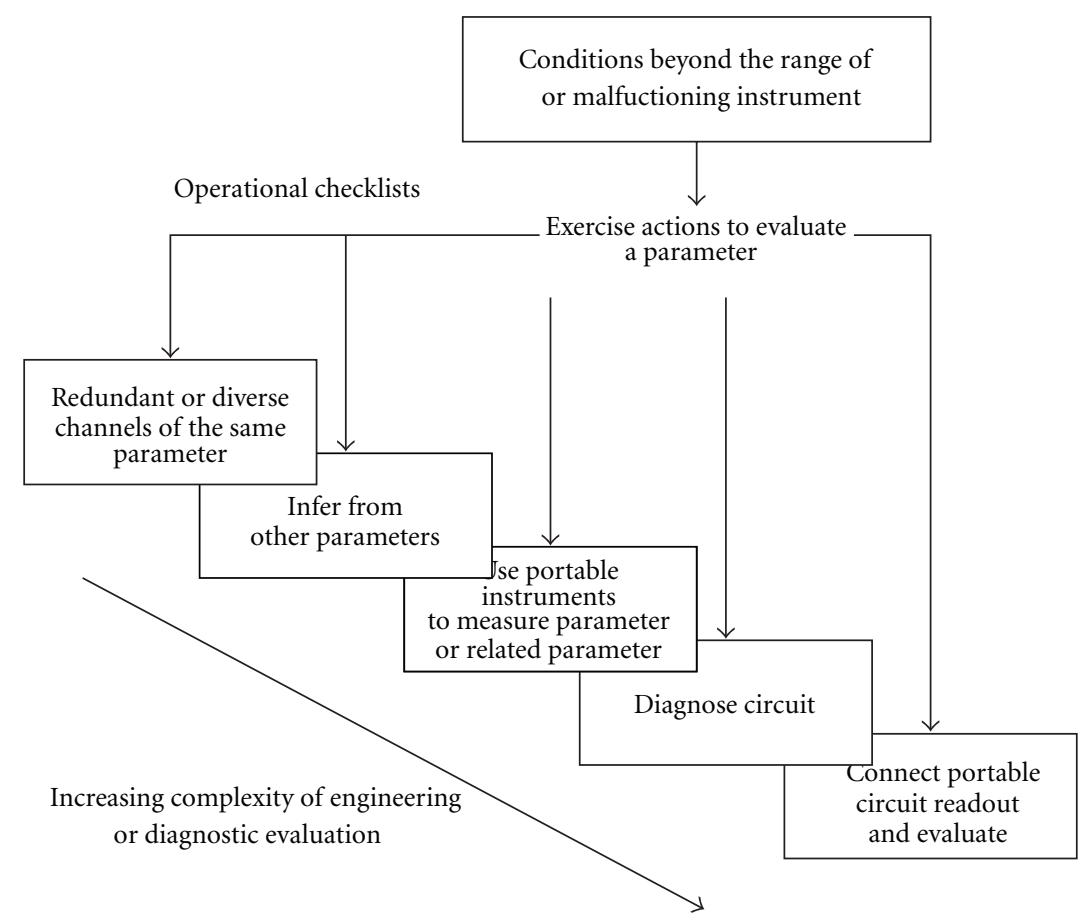

FIGURE 1: Flowchart for the circuit diagnosis methods among the five types of aids.

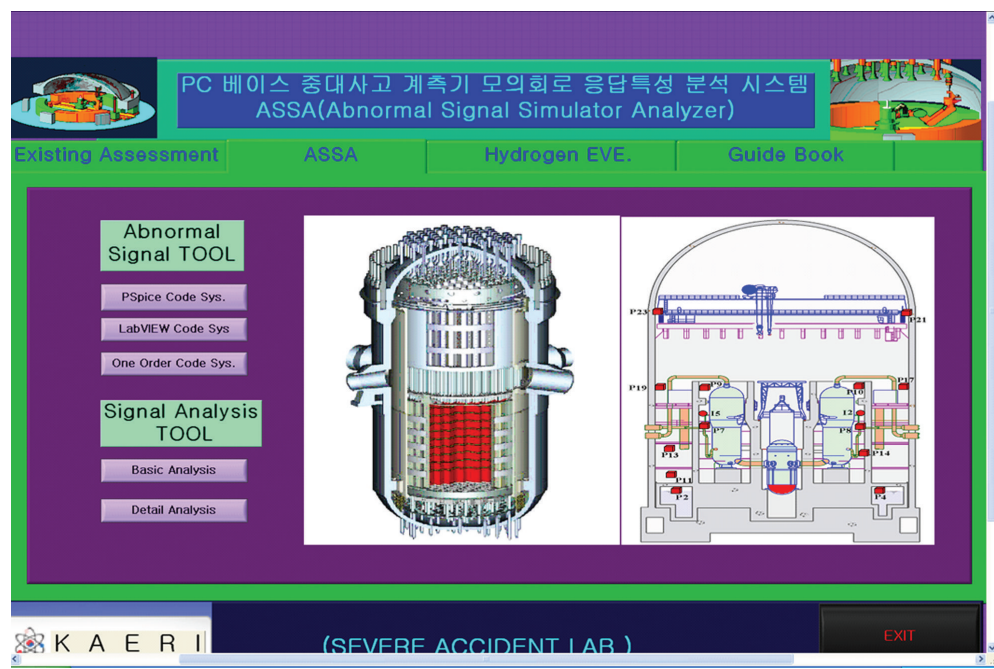

FIGURE 2: Initial screen consisting of functions for ASSA module.

a scenario taken by MAAP4 code. Figure 5 shows a screen for a comparative assessment procedure as the first step for the ASSA module system. As a result, if the proper conditions are not satisfied by using the comparative assessment method, then proceed to a next step as the section of LabVIEW Tool code. As the next step, we need decision making for signal conditions which means three kinds of signal conditions, of which the first is normal condition signals, the second is abnormal condition signals, and the third is out of control condition signals.

In the case of abnormal condition signals, we are interested in the information on the patterns of the abnormal signals and the database for the signal patterns that also could be obtained by the engine code, which then need to be processed by any other five substeps from the analysis tool code, and finally we are able to obtain corrective signals as a final step, but not yet here. There are two codes to be used for the simulation, the designed simulator which is composed of the LabVIEW code as a main analysis tool code, and the PSpice and MultiSIM code as a simulation engine code. For these abnormal signals simulations, a new simulator through an analysis of a typical equivalent circuit modeling has been designed, which is composed of an analysis tool code as a main body and a simulation engine code whose 


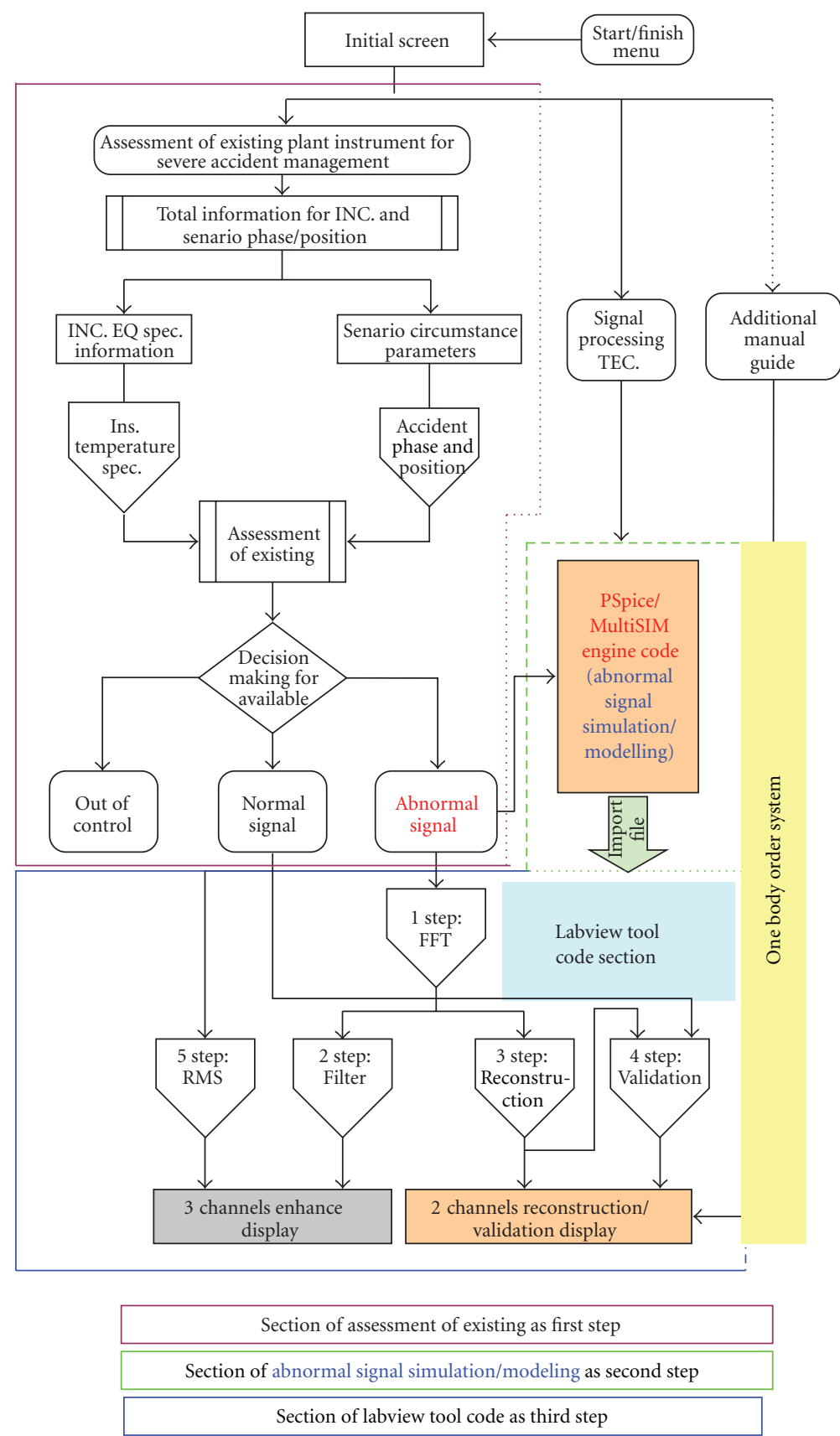

FIGURE 3: Flowchart of the overall design steps for the simulator as ASSA module.

systems are imported to link an output file to an input file.

\section{Detailed Design and Functions of the ASSA Module}

3.1. Functions and Equivalent Circuit of the Engine Code. The engine code for the circuit simulation program was used to simulate the effects of an instrument loop degradation on a measurement condition. This engine code is useful for obtaining a correlation between the simulation circuit conditions and the actual circuit conditions. Although the goal was to develop a real model, the intricacies of developing the appropriate waveforms and incorporating the effects of the induced noise into the circuit turned a degraded circuit loop into lines and lines of netlists. A $4 \sim 20 \mathrm{~mA}$ loop was used to measure the reactor coolant pressure the same as for most instruments. The overall circuit loop consists of a section of a control room, a section of a twisted pair shield cable, and a section of a transmitter. A transmitter circuit was treated as a loop for an important circuit for the passive elements $R-L-C$ which could be affected by the severe accident conditions. In this case, the imported passive elements of the transmitter circuit were $R_{2}, R_{3}, C_{1}$, and $C_{2}$, as distributed parameters 


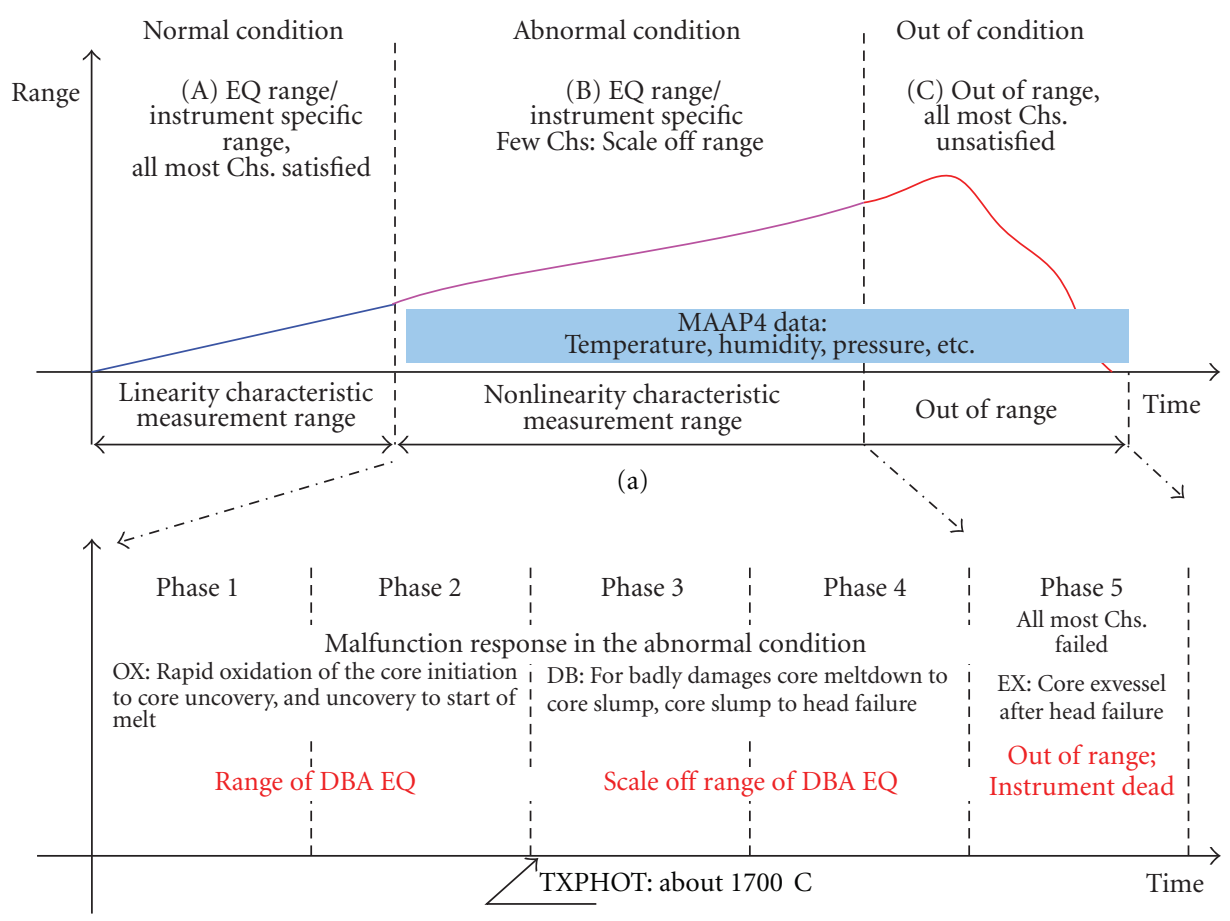

(b)

FIgURE 4: Three EQ conditions and five phases of a scenario taken by MAAP4 code.

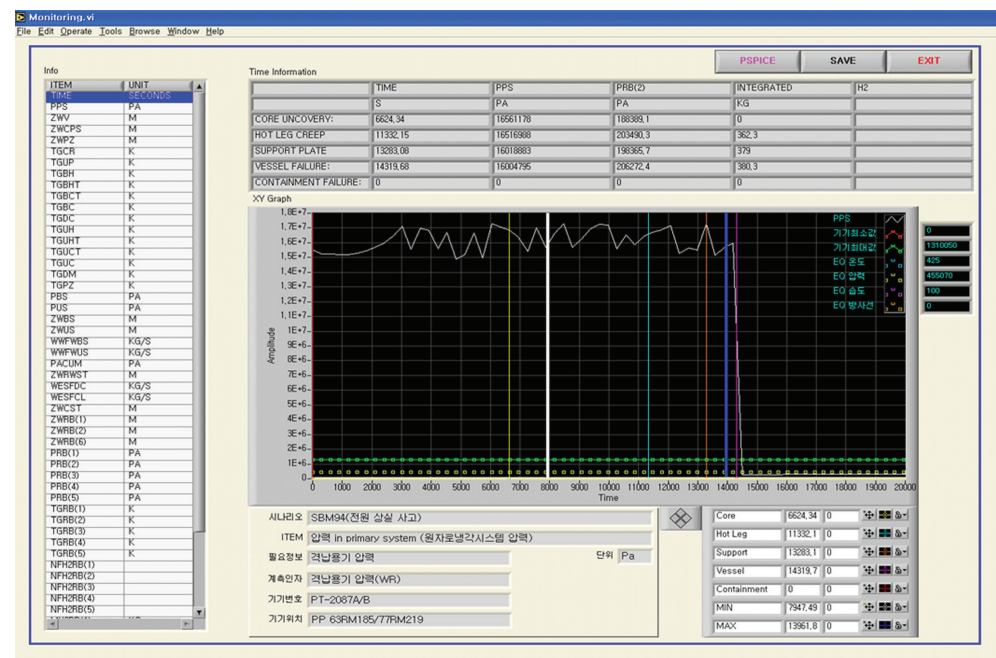

FIGURE 5: Screen of comparative assessment procedure as the first step for the ASSA module.

of the circuit. Figure 6 represents an overall circuit model modified to run in the MultiSIM code.

\subsection{Procedure for a One Body Order Simulation and Digital}

Signal Processing. For the first step, during the operating procedure, the establishment of a simulation circuit modeling by the PSpice/MultiSIM code is attempted, where the PSpice/MultiSIM simulation output file is used for all the files; *.cir, *.sim, and ${ }^{*}$.net files. The next step needs the information on the circuit elements from the ${ }^{*}$.net file, and then the ${ }^{*}$.net file can be controlled by an element in the circuit, for using the Labview * . net file condition, and then we take the csd file out of the ${ }^{*}$.net file in the Labview and execute the system exec.vi file of the PSpice/MultiSIM condition. As the last step, we extract the output results from the csd file, and then the file can be displayed as some of the output abnormal signals. Finally, these signals can be processed by an analyzing tool. Thus, this system will be used for circumstance diagnostic for the abnormal signals, and this code will also allow us to use the enhanced signals from the abnormal signals for some malfunctioning instruments. There are two types of programs which consist 


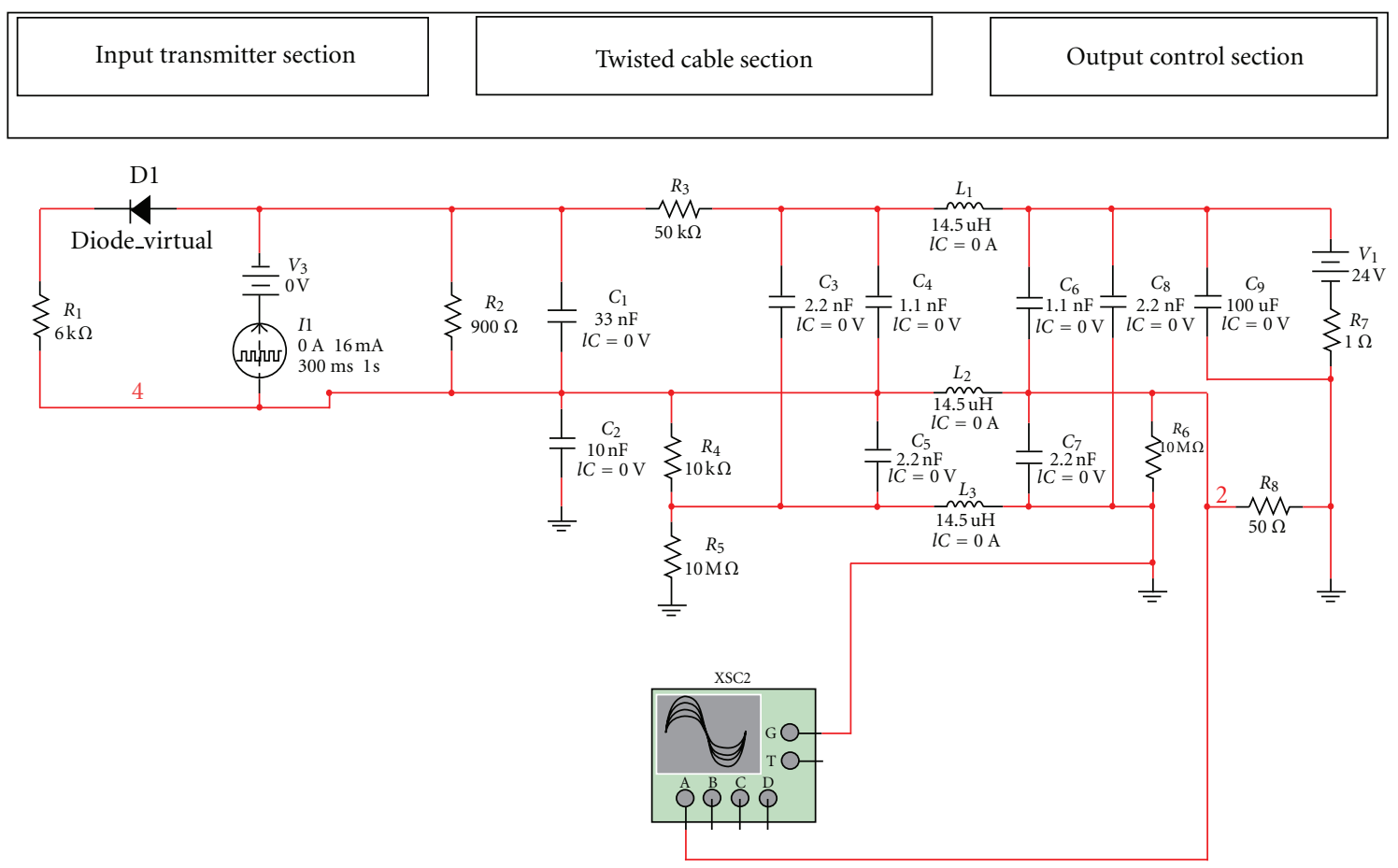

FIGURE 6: Overall circuit model modified to run in the MultiSIM code.

of the PSpice/MultiSIM engine code and the LabVIEW tool code, which can work as a one body order system for this simulation. The overall simulation result data can be used by one operation system as a one order command. So that the programs could be operated quickly and conveniently as an onsite instrument, in this simulation, we changed the element values in the circuit which is possible by directly changing the $R-C$ element value according to the temperature condition in the initial screen menu of the tool code, so we do not need to use the functions of the engine code.

As a result, it is also possible to change the output signal patterns according to the changing element values in the tool code menu functions under SACs. It is also easy and convenient for a signal analysis for some types of noise patterns. The output results can be extended and analyzed for their response characteristics by the tool code, because the LabVIEW code has powerful analyzing functions and good interface while being onsite. Figure 7 shows the block diagram for a one body order simulation system. The loop circuit was composed of three sections which include the pressure transmitter section, the twisted shielded pair connection cable section, and the receiver section. The pressure transmitter has been simplified to obtain an equivalent representation of the resisters, capacitors, and inductors as distributive parameters of the circuit. It also includes voltage or current sources that can be varied through the circuit simulation code to obtain realistic circuit response characteristics from environmental changes in the containment. A specific parameter includes: an input leakage (shunt) resistance that is affected by damage to an interconnecting cable and; input series resistance to represent the

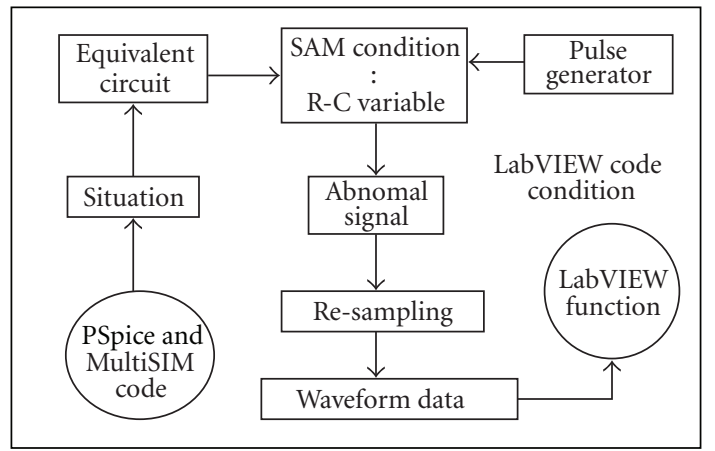

Figure 7: Block diagram for one body order simulation system.

spliced connections that might be affected by corrosion and whiskers phenomenon. Input capacitance is increased easily by a water intrusion into the cable or the transmitter housing. Capacitance for the ground is easily increased by the presence of water. A diode and load resistors represent the power supply current. A current generator represents the actual transmitter output signal. A typical cable is represented to describe the wire inductance and resistance, and the dielectric capacitance. The shield is represented as a third conductor. It is simplified to eliminate all the resistances, and to only model the inductance and capacitance. The voltage source drives a current to a voltage sensing resistor in series with the transmitter. Negative sides of the voltage source and the shields are attempted for the ground source in this analysis. Figure 8 shows a detail block diagram for the signal processing and analysis from the ASSA module system. Figure 9 is a screen of the ASSA for one body order simulation system. 


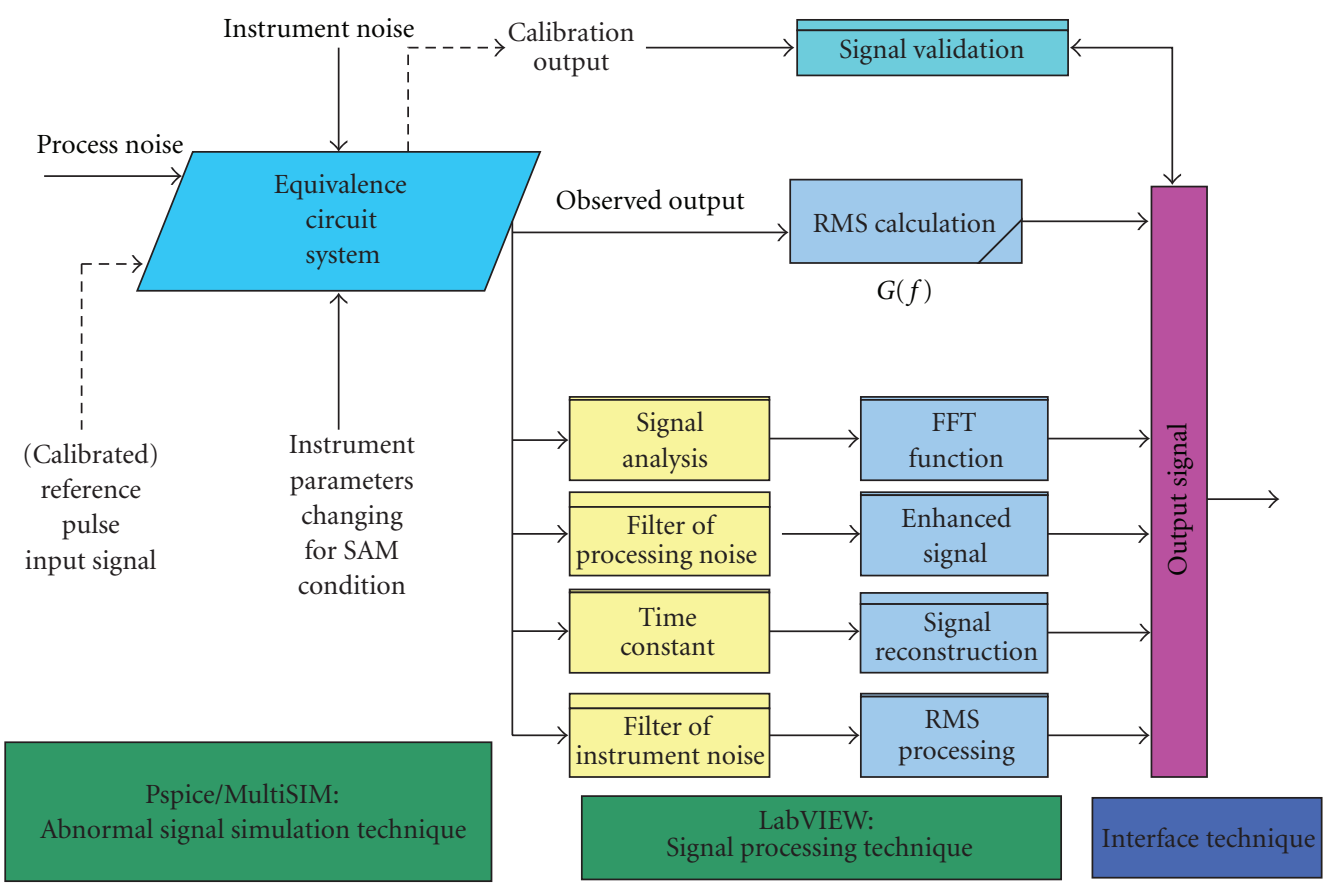

FIGURE 8: Block diagram for the signal processing and analysis from ASSA module system.

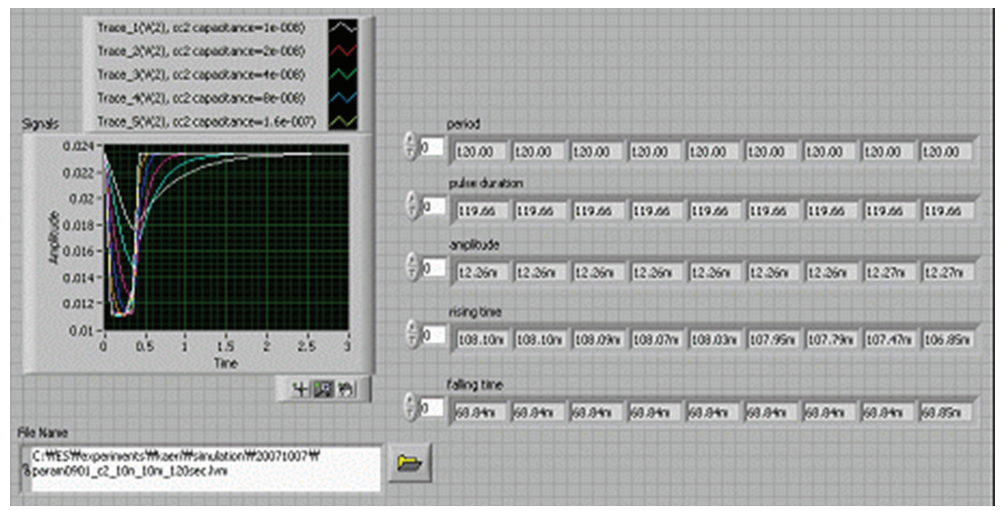

FIgURE 9: A screen of the ASSA for a one body order simulation system.

\section{Simulation and Response Characteristic Analysis from the Obtained Data}

There are two types regarding information of pulse parameters which means changing a value for the output pulse signal with certain information to diagnosis a circuit condition such as a voltage level, a time constant including information of a rising time, falling time, and bandwidth compare to information for pulse parameters of the reference input signal. As a result, when we can analyze the cause of an equivalent circuit, which consists of an $R-L-C$ distribution parameter, for a $4 \sim 20 \mathrm{~mA}$ instrument of a severe accident condition from response characteristics, it is also the same meaning that is also possible to detect and diagnose the abnormal signal of a malfunctioning instrument from a simulation and response characteristic analysis. Figure 10 is patterns of output pulse signal of ASSA for a one body order simulation system.

Method for the pulse parameter estimation: for pulse parameter, estimation for ASSA module has designed as the basic concept for circuit analysis procedure. In addition to this method, there is pulse detection logic, an abnormal pulse filter, and a pulse parameter estimation for pulse parameter estimation was already said and it is redundant. The pulse detection logic is operable to detect pulses and to estimate a pulse parameter of each detected pulse [10]. The abnormal pulse filter is operable to analyze a pulse parameter of the detected pulses and to determine whether there are any abnormal pulses based on the analysis. The pulse parameter estimation is operable to estimate a pulse parameter for 


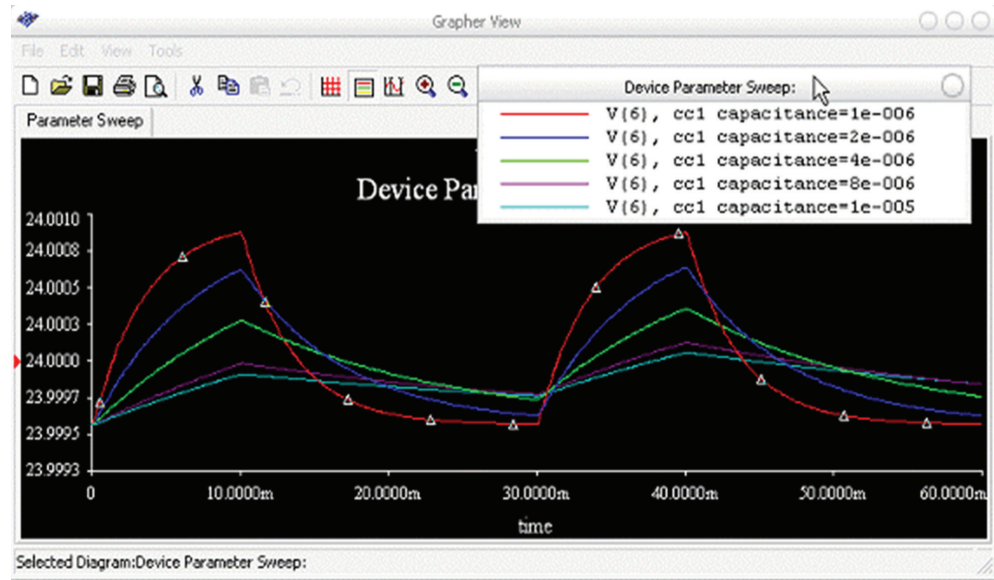

FIGURE 10: Patterns of output pulse signal of ASSA for a one body order simulation system.

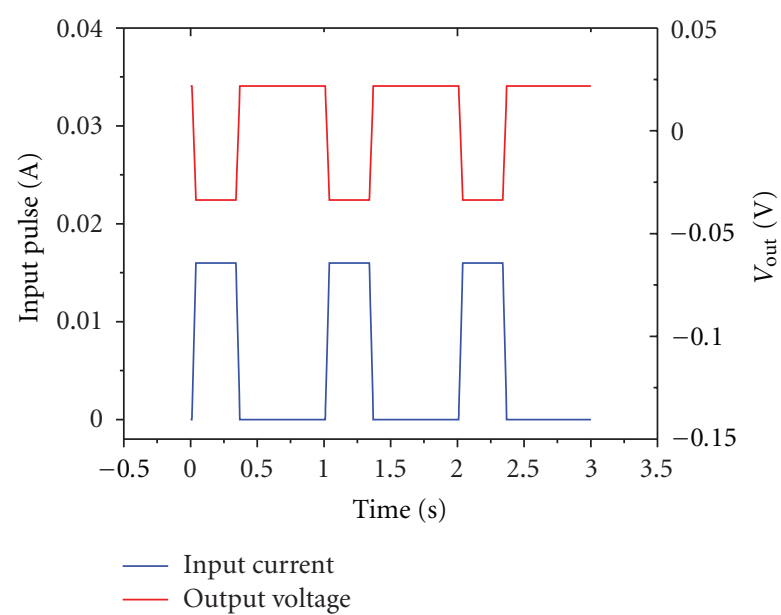

FIGURE 11: Reference input current (A) and output voltage (V) data for the pulse parameter as an initial value.

the signal based on an analysis of the detected pulses and information pertaining to there being any abnormal pulses in the signal.

4.1. Defining the Reference Signal from the Equivalent Circuit. Figure 11 is reference input current (A) and output voltage (V) data for the pulse parameter as an initial value. This reference pulse signal was defined in the engine code as a linear approximation, with a $0-16 \mathrm{~mA}$ pulse with a $0 \mathrm{~ms}$ delay, $30 \mathrm{~ms}$ rising time, $30 \mathrm{~ms}$ falling time, and a 1 PPS repetition rate. The reference pulse also describes an output current pulse as measured across the current to voltage converter at the receiver. This is a reference input current $I$ (A) and output voltage $V(\mathrm{~V})$ data of the pulse parameter as an initial value. In this figure, it appears as about 0.1 to 1.0 (V) pulse at a temperature of $27\left({ }^{\circ} \mathrm{C}\right)$.

4.2. Results with Change of Resistances. Figures 12 and 13 show the output voltage level of the pulse parameter according to a change of the resistance $R_{2}$ which range has

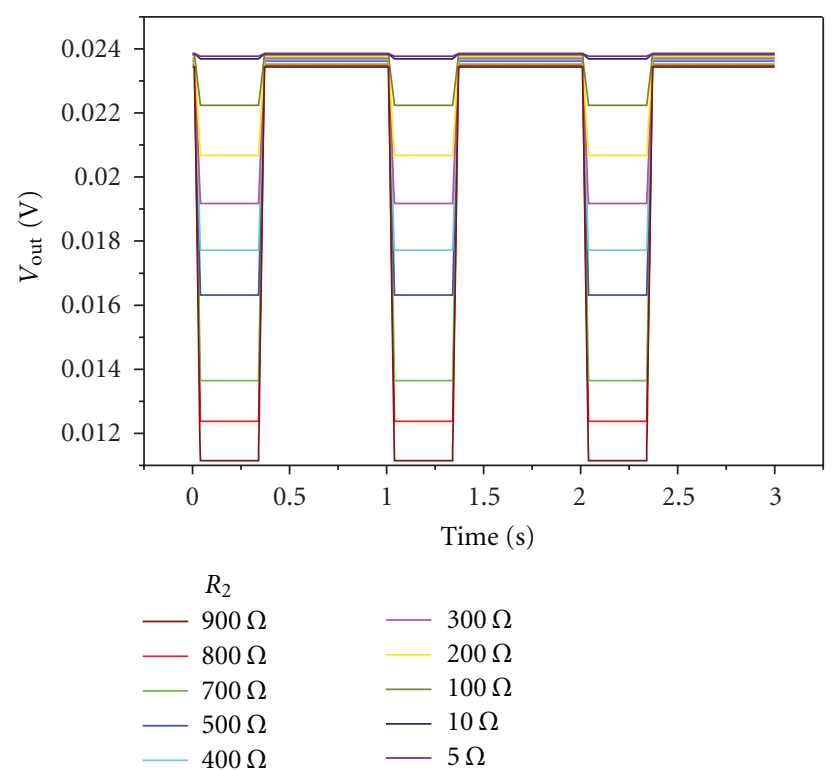

FIGURE 12: Output voltage levels for the pulse parameter for a change of the resistance of $R_{2}(5(\Omega) \sim 900(\Omega))$.

$5(\Omega) \sim 900(\Omega)$ and $1(\mathrm{k} \Omega) \sim 10(\mathrm{k} \Omega)$, respectively. Figure 14 shows the variations of output voltage according to the $R_{2}$. In the case of the $R_{2}$ simulation using the tool code, it can be seen that the resistor value changes for the high-voltage level data which means a good linearity (sensitivity) response characteristic over $1(\mathrm{k} \Omega)$ to $10(\mathrm{k} \Omega)$. In this resistance $R_{2}$, the impulse response of an equivalent circuit for the 4-20 mA is its output when presented with an input standard signal called an impulse. The impulse response refers to the reaction of the circuit system which was consisted with that resistance $R_{2}$ to be obtain the response characteristic for the maximum resistance changing value facing each external temperature as the changing parameter, the response characteristic has an increasing characteristic in the circuit according to basic circuit theory, because the resistor $\left(R_{2}\right)$ was consisted of the parallel component. 


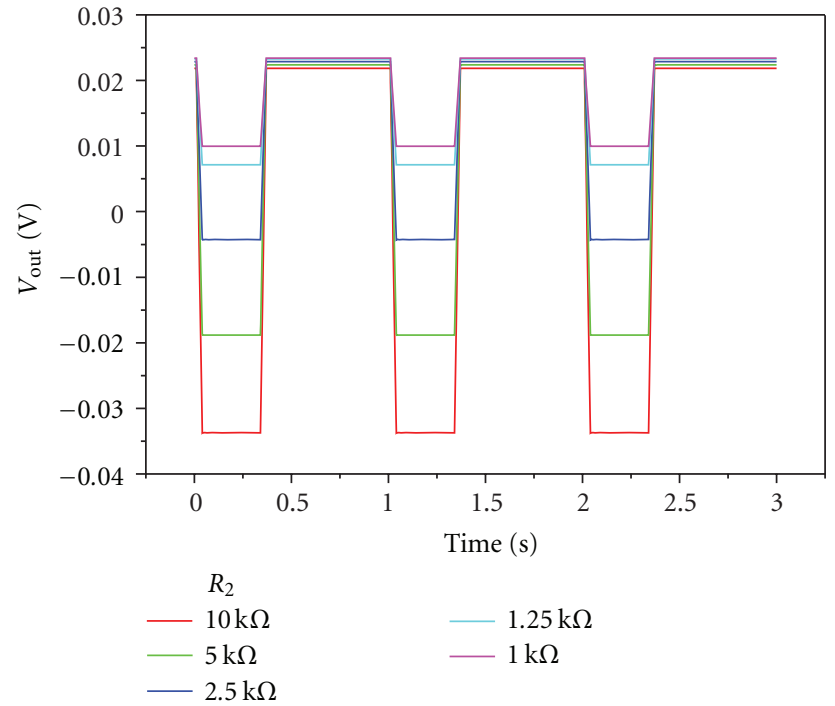

Figure 13: Output voltage levels for the pulse parameter for a change of the resistance of $R_{2}(1(\mathrm{k} \Omega) \sim 10(\mathrm{k} \Omega))$.

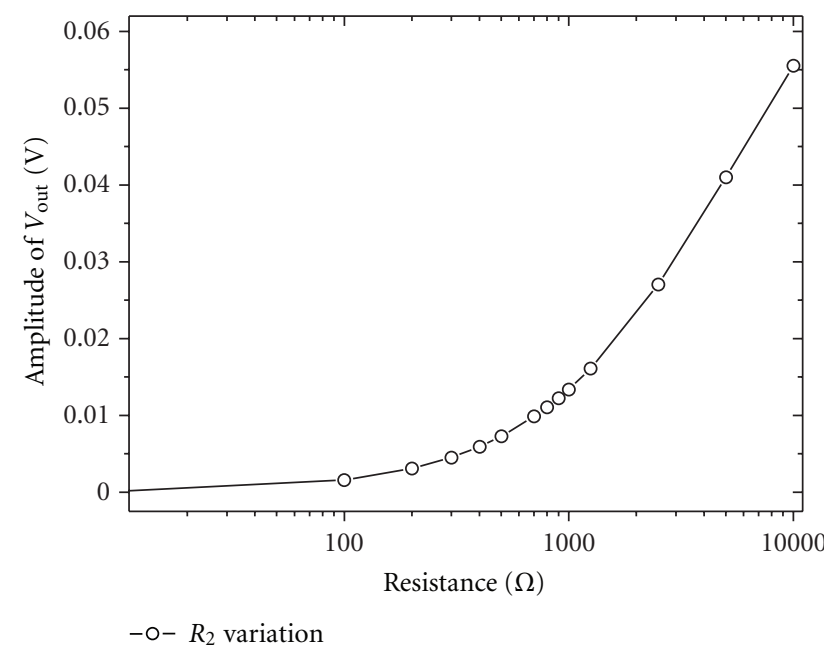

FIGURE 14: Response characteristic of the output voltage for the pulse parameter for a change of the resistance of $R_{2}(5(\Omega) \sim 10$ $(\mathrm{k} \Omega)$ ).

Figures 15 and 16 show the output voltage level of the pulse parameter according to a change of the resistance $R_{3}$ which has a range of $5(\Omega) \sim 4(\mathrm{k} \Omega)$ and $5(\mathrm{k} \Omega) \sim$ $50(\mathrm{k} \Omega)$, respectively. In the case of the $R_{3}$ simulation using the tool code, it can be seen that the resistor value changes for the high voltage level data which means a good linearity (sensitivity) response characteristic over $5(\Omega)$ to $50(\mathrm{k} \Omega)$, in the case of an overall response characteristic with a decreasing response characteristic for an increasing resistance because the resistor $\left(R_{3}\right)$ was consisted of the serial component in Figure 17 according to the basic circuit theory. Figure 17 shows a response characteristic of the output voltage for the pulse parameter for a change of the resistance of $R_{3}(5(\Omega) \sim 50(\mathrm{k} \Omega))$.

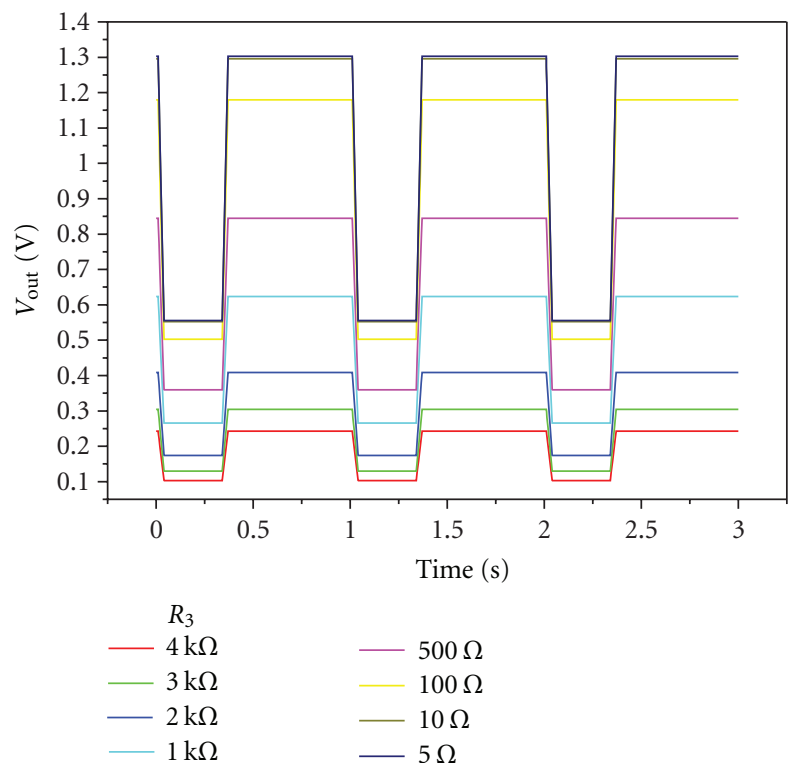

Figure 15: Output voltage levels for the pulse parameter for a change of the resistance of $R_{3}(5(\Omega) \sim 4(\mathrm{k} \Omega))$.

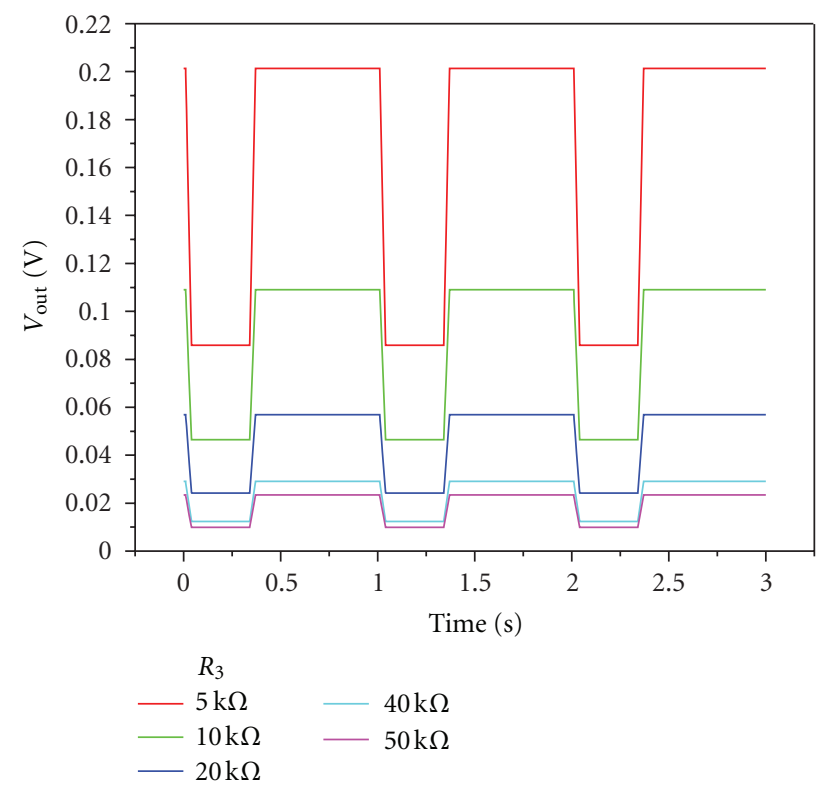

FIgURE 16: Output voltage levels for the pulse parameter for a change of the resistance of $R_{3}(5(\mathrm{~K} \Omega) \sim 50(\mathrm{~K} \Omega))$.

4.3. Results with Change of Capacitances. Figure 18 shows the output voltage level data and the time constant for the pulse parameter according to a change of the capacitance of $C_{1}$ which has a range of $33(\mathrm{nF}) \sim 33(\mathrm{mF})$. Figure 19 shows the response characteristics of the output voltage curve for the pulse parameter according to a change of the capacitance of $C_{1}$. Figure 20 shows the response characteristics of the rising time ( $\operatorname{Tr}$ ) for the pulse parameter according to a change of the capacitance of $C_{1}$. In the case of the $C_{1}$ simulation by using the tool code, it can be seen that the capacitance 


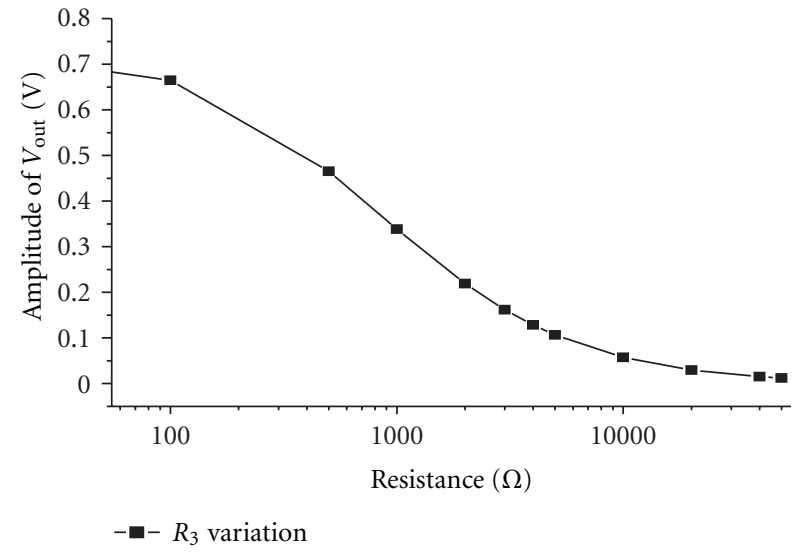

FIGURE 17: Response characteristic of the output voltage for the pulse parameter for a change of the resistance of $R_{3}(5(\Omega) \sim 50$ $(\mathrm{k} \Omega)$ ).

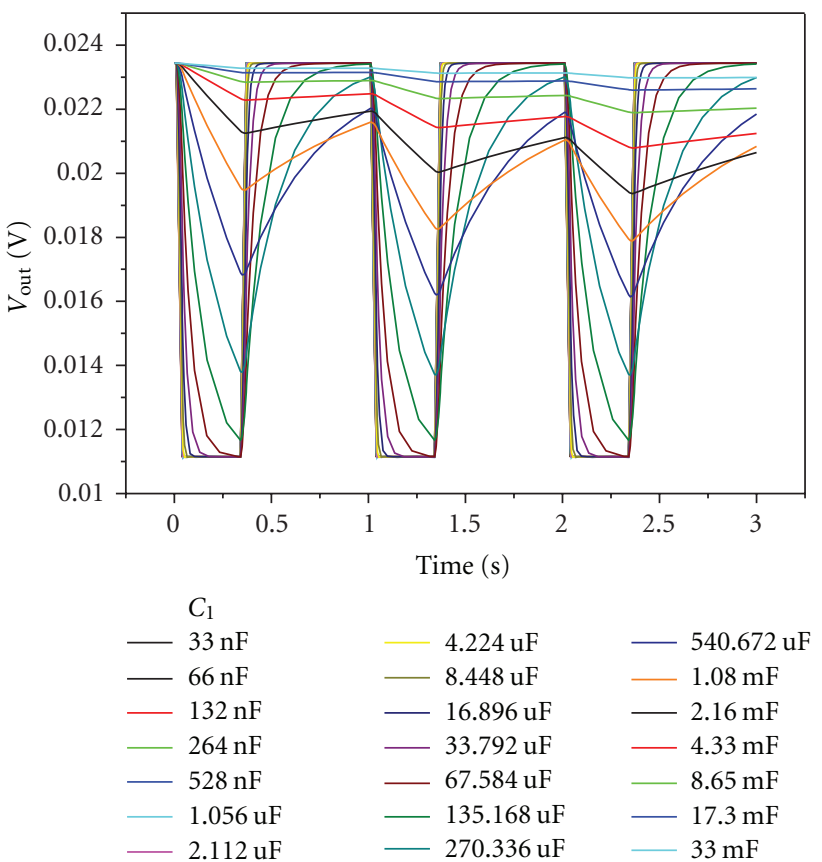

FIGURE 18: Output data for the pulse parameter for a change of the capacitance of $C_{1}(33(\mathrm{nF}) \sim 33(\mathrm{mF}))$.

value changes for the high voltage level data which means a good linearity (sensitivity) response characteristic over the same range, in the case of an overall response characteristic with a decreasing response characteristic for an increasing capacitance. The response characteristic of the rising time (Tr) for the pulse parameter which also means a good linearity (sensitivity) for the response characteristics over the same range, in the case of an overall response characteristic, reveals an increasing response characteristic for an increasing capacitance of $C_{1}$. In this capacitance $C_{1}$, the impulse response of an equivalent circuit for the $4-20 \mathrm{~mA}$ is its output when presented with an input standard signal called an impulse. The impulse response refers to the reaction of

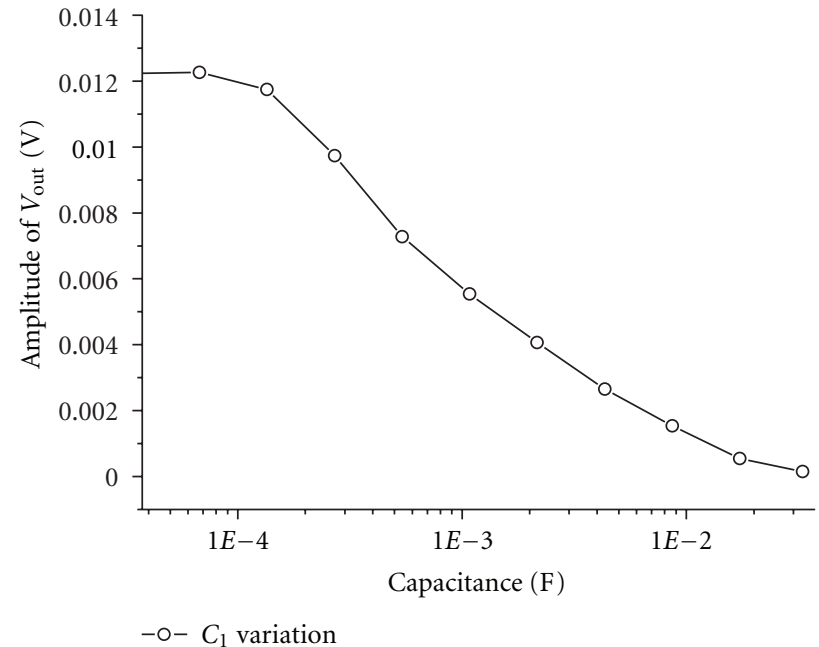

FIGURE 19: Response characteristic of the output voltage curve for the pulse parameter for a change of the capacitance $C_{1}(33(\mathrm{nF}) \sim 33$ $(\mathrm{mF}))$.

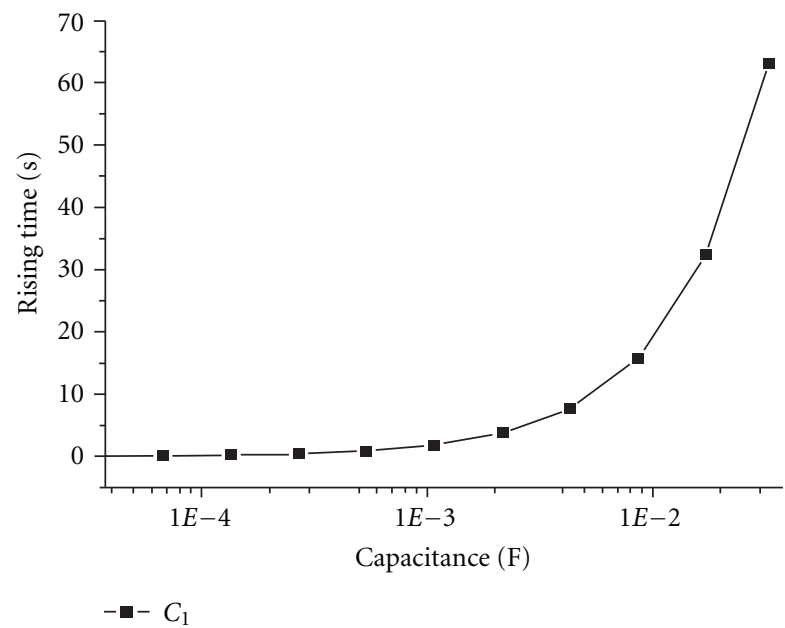

FIGURE 20: Response characteristic of the rising time ( $\mathrm{Tr}$ ) for the pulse parameter for a change of the capacitance of $C_{1}(33(\mathrm{nF}) \sim 33$ $(\mathrm{mF}))$.

the circuit system which was consisted with that capacitance $C_{1}$ to obtain the response characteristic for the maximum capacitance changing value facing each external temperature as the changing parameter, the response characteristic of the output voltage curve is a decreasing characteristic, the response characteristic of the rising time (Tr) is an increasing characteristic in the circuit according to basic circuit theory, because the capacitance $\left(C_{1}\right)$ was consisted of the parallel component.

Figure 21 shows the output voltage level data for the pulse parameter according to a change of the capacitance of $C_{2}$ which has a range of $10(\mathrm{nF}) \sim 10(\mathrm{mF})$. Figure 22 shows the response characteristics of the output voltage level data for the pulse parameter according to a change of the capacitance of $C_{2}$. Figure 23 shows the response characteristics of the rising time ( $\operatorname{Tr}$ ) for the pulse parameter according to a change 


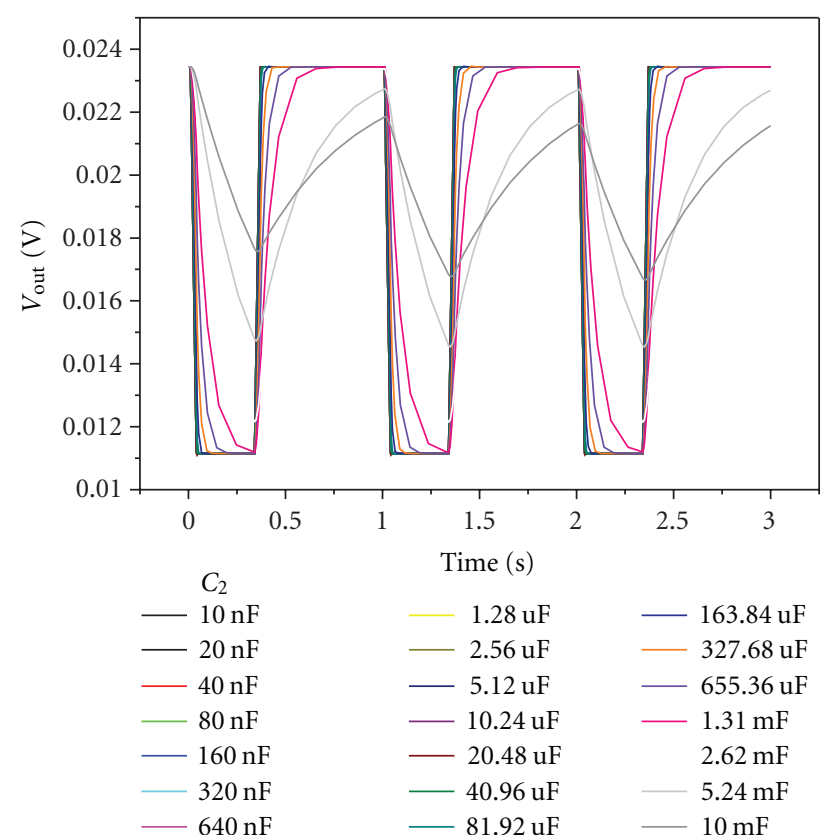

FIgURE 21: Output data for the pulse parameter for a change of the capacitance of $C_{2}(10(\mathrm{nF}) \sim 10(\mathrm{mF}))$.

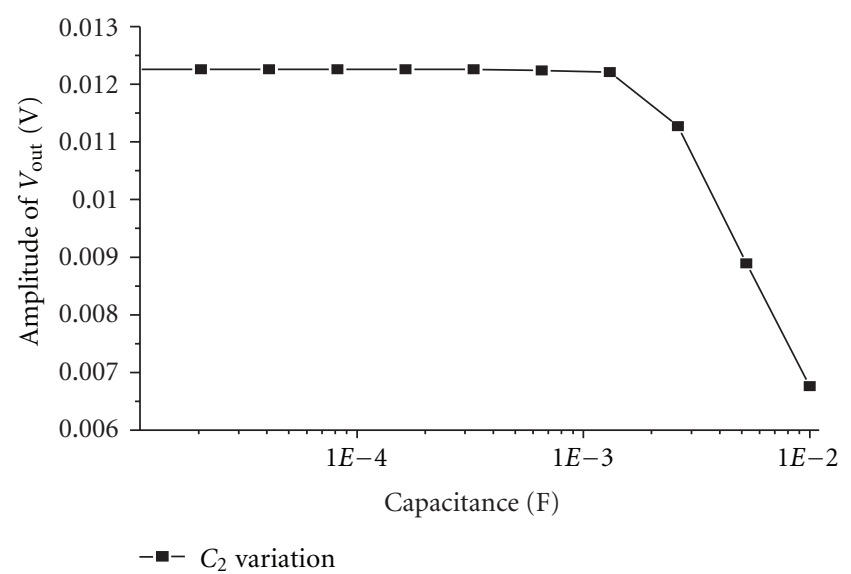

Figure 22: Response characteristic of the output voltage for the pulse parameter for a change of the capacitance of $C_{2}(10(\mathrm{nF}) \sim 10$ $(\mathrm{mF}))$.

of the capacitance of $C_{2}$. In the case of the $C_{2}$ simulation using the tool code, it can be seen that the capacitance value changes for the high voltage level data which means a good linearity (sensitivity) response characteristic over the same range, in the case of an overall response characteristic with a decreasing response characteristic for an increasing capacitance at $1.31(\mathrm{mF})$, until about $1.31(\mathrm{mF})$ it was a steady state.

But the response characteristic of the rising time ( $\mathrm{Tr}$ ) for the pulse parameter which means a good linearity (sensitivity) for the response characteristics over the same range, in the case of an overall response characteristic reveals an increasing response characteristic for an increasing

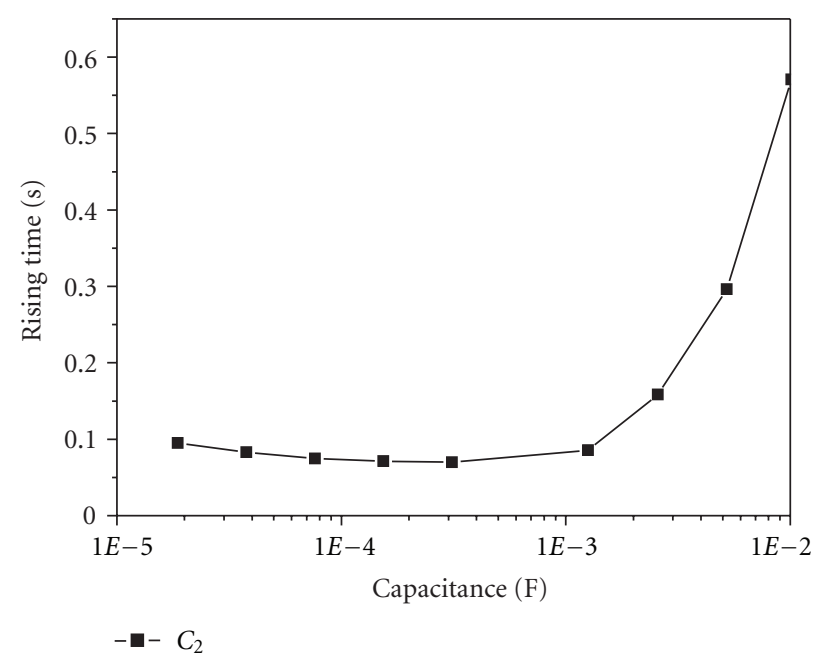

FIGURE 23: Response characteristic of the rising time for the pulse parameter for a change of the capacitance of $C_{2}(10(\mathrm{nF}) \sim 10(\mathrm{mF}))$.

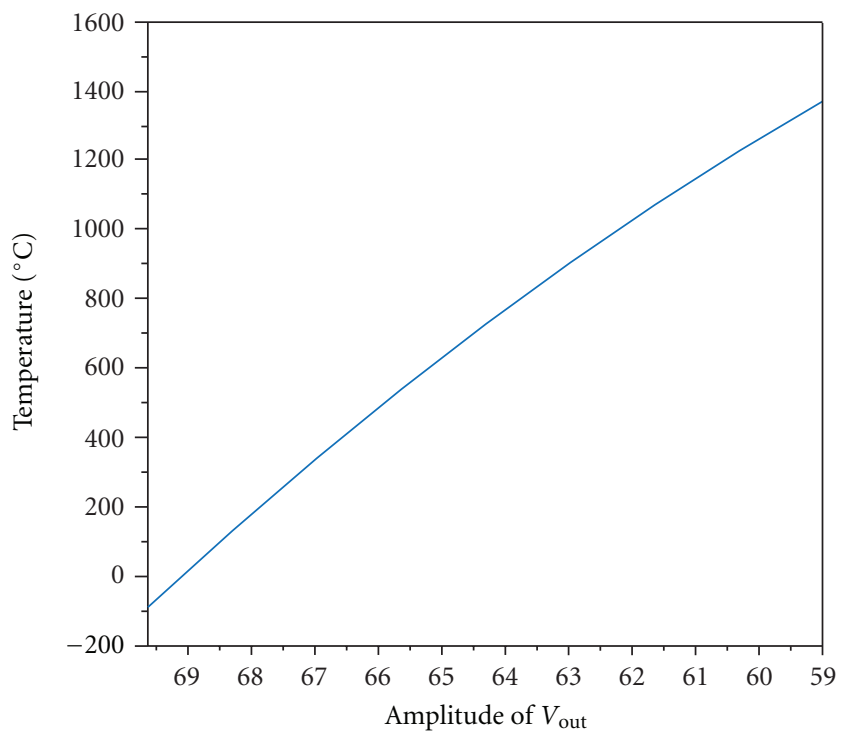

FIgure 24: Relation curve between the $T$ temperature and the Amplitude of $V_{\text {out }}$ according to (1).

capacitance of $C_{2}$ in the circuit according to basic circuit theory.

\section{Correlation Equation}

5.1. Correlative Equation between $R_{2}$ and Temperature. There is a correlative equation between the temperature and the amplitude of an output voltage in the equivalent circuit, which means that $R_{2}$ has been changing in the resistance range as a distributed parameter when the $T$ temperature has been changing in the temperature range due to the accident conditions. Equation (1) is a correlative equation between the temperature and the amplitude of an output voltage. Figure 24 shows a relation curve between the $T$ temperature and the amplitude of the $V_{\text {out }}$ according to (1). From this 


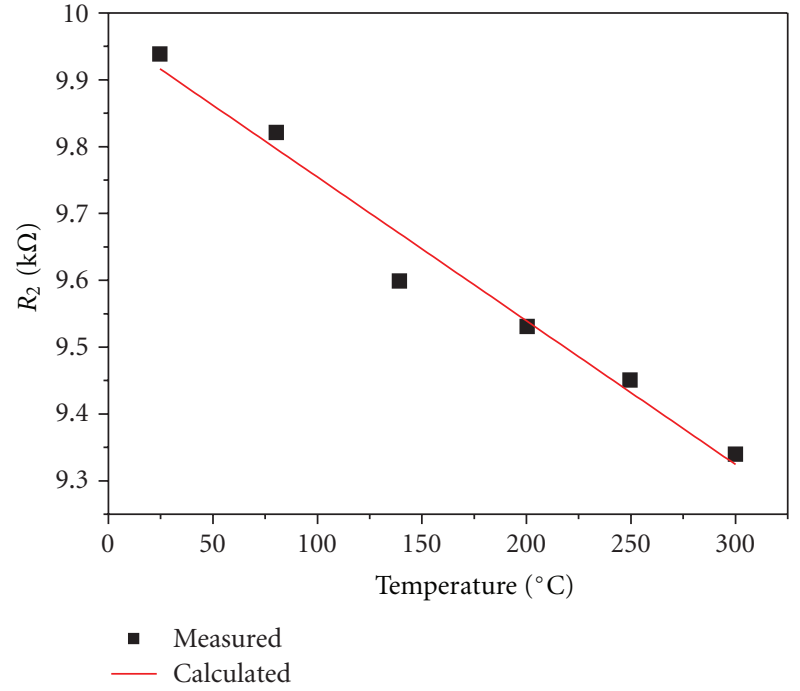

Figure 25: Relation curve between $R_{2}$ and the $T$ temperature according to (2).

equation, for an inverse operation, it could also be said that $T$ temperature has been changing in the temperature range when $R_{2}$ has been changing in the resistance as a distributed parameter in the equivalent circuit due to the accident conditions. In this case of the changing $R_{2}$ resistance value according to the changing temperature, experimental data is obtained by using in an electric oven as a real test. Equation (2) is also a correlative equation between the temperature and the amplitude of an output voltage, in the case of the changing $R_{2}$ resistance according to the $T$ temperature. Figure 25 shows the relation curve between the $R_{2}$ and the $T$ temperature according to (2). Table 1 shows the changing $R_{2}$ resistance range according to the temperature changing range in a real test using in an electric oven:

$$
T=\frac{\left(\left(1 /\left(R_{3}+R_{7}+R_{8}\right)\right)\left(i_{m} R_{8} / v_{0}-1\right)-1 / R_{1}\right)^{-1}-R_{0}}{c_{1}}+T_{0},
$$

$$
R_{2}(T)=c_{1}\left(T-T_{0}\right)+R_{0},
$$

where $T$ : changing temperature, $T_{0}$ : normal/initial temperature, $R_{0}$ : normal/initial resistance, $R_{2}(T)$ : changing resistance at $T$ temperature, $c_{1}: 1$ st-order temperature coefficient, $i_{m}$ : maximum input pulse current, $v_{0}$ : the normal/initial voltage, and $R_{3}, R_{7}, R_{8}$ : the circuit elements.

5.2. Relation between the $C_{1}$ and the Time Constant. The changing range for the time constant of the output voltage in the equivalent circuit when the $C_{1}$ has a maximum changing range from $33 \mathrm{nF}$ to $33 \mathrm{mF}$, to be placed in extreme simulation conditions should be investigated. From the simulation result, it is also possible to explain an approximate equation for the relation between the $C_{1}$ and the time constant. In the case of above $33 \mathrm{uF}$, the time constant of the output voltage has a response characteristic as a steep
TABLE 1: $R_{2}$ Resistance changing range according to the temperature changing range in a real test.

\begin{tabular}{lc}
\hline$T\left({ }^{\circ} \mathrm{C}\right)$ & $R_{2}(\mathrm{k} \Omega)$ \\
\hline 25 & 9.94 \\
80 & 9.82 \\
140 & 9.60 \\
200 & 9.53 \\
250 & 9.45 \\
300 & 9.34 \\
\hline
\end{tabular}

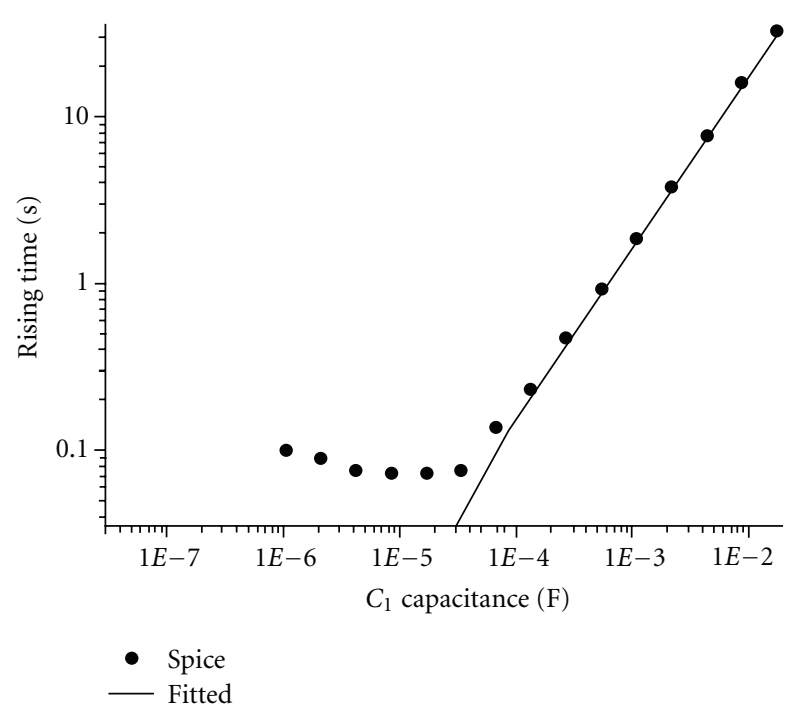

FIGURE 26: A linear response characteristic between the rising time and $C_{1}$.

slope but it has response characteristic of almost no change when the $C_{1}$ has a changing range from $33 \mathrm{nF}$ to $33 \mathrm{uF}$. Therefore, the time constant of the output voltage has a linear response characteristic when the $C_{1}$ has a changing range from $33 \mathrm{uF}$ to $33 \mathrm{mF}$. Figure 26 shows a linear response characteristic between the rising time parameter and when $C_{1}$ has a changing range from $33 \mathrm{uF}$ to $33 \mathrm{mF}$. Equation (3) defines a relation between the $C_{1}$ and the time constant as a rising time parameter from an output voltage shape. When the $t$ is a time constant as a rising time parameter, $c$ is the 1st-order temperature coefficient for the $C_{1}$ changing range. To obtain a rising time for $R$ - $C$ elements, Figure 27 shows changing output data for the pulse parameter for a changing range of the temperature of $C_{2}\left(27\left({ }^{\circ} \mathrm{C}\right) \sim 87\left({ }^{\circ} \mathrm{C}\right)\right)$ according to correlation equation temperature coefficient to the time constant as a rising time parameter for $R$ - $C$ elements. Table 2 is output parameters of the pulse parameter according to a change of the capacitance of $C_{1}$ :

$$
t=1682 c-0.0155417,
$$

where $t$ : time constant as a rising time parameter, $c$ : 1 st-order temperature coefficient. 
TABLE 2: Output data of the pulse parameter according to a change of the capacitance of $C_{1}$.

\begin{tabular}{|c|c|c|c|}
\hline$C_{1}$ (Parameter) & Amplitude & Rising time & Falling time \\
\hline $33 n$ & $1.2240 E-02$ & $1.08 E-01$ & $6.88 E-02$ \\
\hline $66 n$ & $1.2240 E-02$ & $1.08 E-01$ & $6.88 E-02$ \\
\hline $132 n$ & $1.2240 E-02$ & $1.07 E-01$ & $6.88 E-02$ \\
\hline $264 n$ & $1.2240 E-02$ & $1.06 E-01$ & $6.88 E-02$ \\
\hline $528 \mathrm{n}$ & $1.2250 E-02$ & $1.04 E-01$ & $6.88 E-02$ \\
\hline $1.056 \mathrm{u}$ & $1.2250 E-02$ & $9.95 E-02$ & $6.88 E-02$ \\
\hline $2.112 \mathrm{u}$ & $1.2240 E-02$ & $8.95 E-02$ & $6.88 E-02$ \\
\hline $4.224 \mathrm{u}$ & $1.2250 E-02$ & $7.58 E-02$ & $6.88 E-02$ \\
\hline $8.448 \mathrm{u}$ & $1.2250 E-02$ & $7.27 E-02$ & $6.88 E-02$ \\
\hline $16.896 \mathrm{u}$ & $1.2240 E-02$ & $7.20 E-02$ & $6.88 E-02$ \\
\hline $33.792 \mathrm{u}$ & $1.2240 E-02$ & $7.48 E-02$ & $8.35 E-02$ \\
\hline $67.584 \mathrm{u}$ & $1.2260 E-02$ & $1.37 E-01$ & $1.41 E-01$ \\
\hline $135.168 \mathrm{u}$ & $1.1740 E-02$ & $2.33 E-01$ & $2.15 E-01$ \\
\hline $270.336 \mathrm{u}$ & $9.7300 E-03$ & $4.67 E-01$ & $2.57 E-01$ \\
\hline $540.672 \mathrm{u}$ & $7.2800 E-03$ & $9.28 E-01$ & $2.67 E-01$ \\
\hline $1.08 \mathrm{~m}$ & $5.5400 E-03$ & 1.86 & $2.68 E-01$ \\
\hline $2.16 \mathrm{~m}$ & $4.0600 E-03$ & 3.77 & $2.68 E-01$ \\
\hline $4.33 \mathrm{~m}$ & $2.6500 E-03$ & 7.71 & $2.70 E-01$ \\
\hline $8.65 \mathrm{~m}$ & $1.5400 E-03$ & 15.82 & $2.73 E-01$ \\
\hline $17.3 \mathrm{~m}$ & $5.4459 E-04$ & 32.39 & $2.74 E-01$ \\
\hline $33 \mathrm{~m}$ & $1.4995 E-04$ & 63.16 & $2.79 E-01$ \\
\hline
\end{tabular}

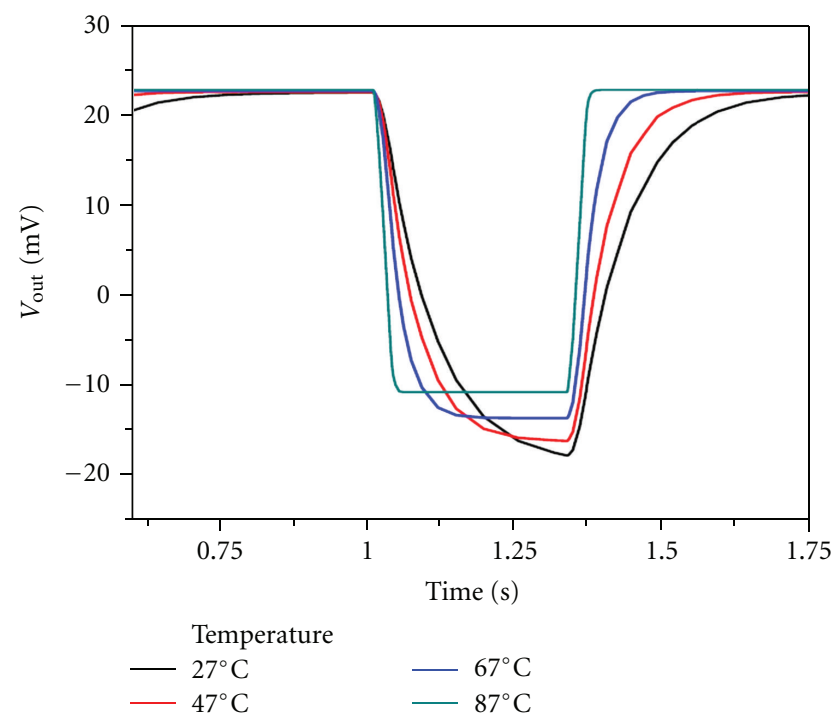

FIgURE 27: Output data for the pulse parameter for a change of the temperature of $C_{2}\left(27\left({ }^{\circ} \mathrm{C}\right) \sim 87\left({ }^{\circ} \mathrm{C}\right)\right)$.

5.3. Data Analysis. We could obtain frequency response characteristics of the multidimensions from the pulse parameter for a changing range of the capacitance $C_{1}$. From this Fourier fast transform (FFT) analysis of the 3 dimensions plot, it will find the activity of distorting shape and it become loosing for a direct current (DC) property for a changing range. Therefore, it also will be able to provide correct

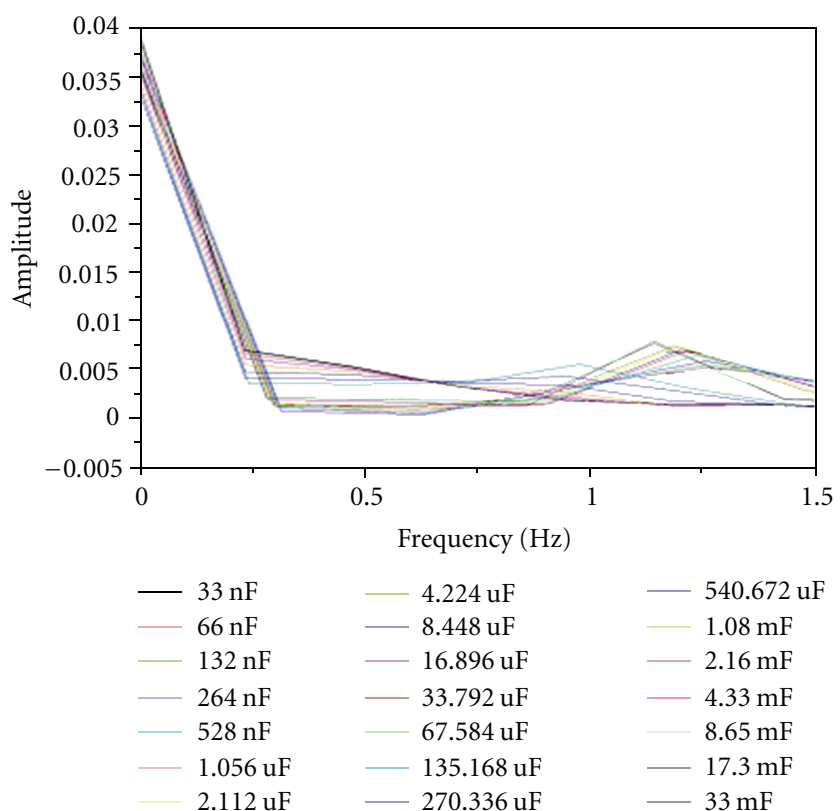

FIGURE 28: Frequency response characteristic of two dimensions of the pulse parameter for a change of the capacitance $C_{1}$.

fault detection and diagnosis of the faulted components through the monitoring system for error signal detection and diagnosis. Figures 28 and 29 show frequency response characteristic each of two dimensions and three dimensions 


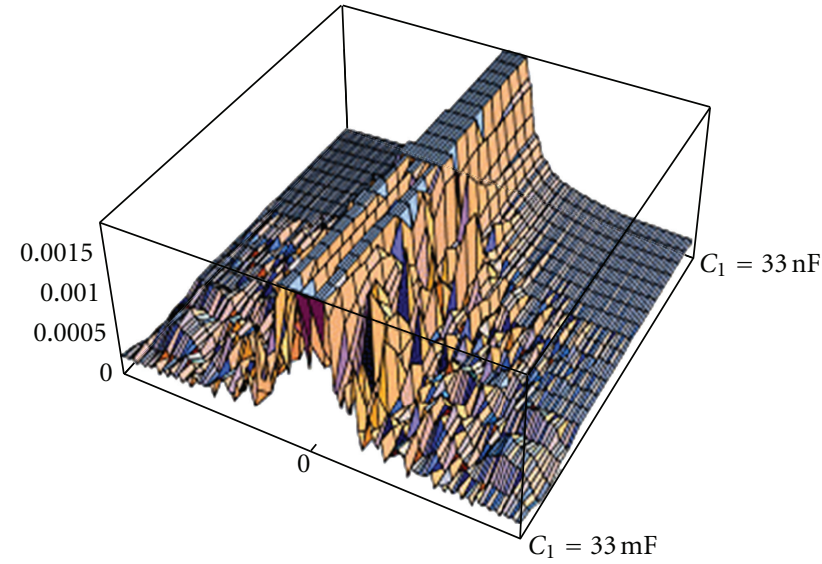

FIGURE 29: Frequency response characteristic of three dimensions of the pulse parameter for a change of the capacitance $C_{1}$.

of the pulse parameter for a change of the capacitance $C_{1}$, respectively.

\section{Conclusions}

In this paper, we provided a new simulator (called ASSA) developed through a circuit simulation with instrument diagnosis methods. Its underlying design requirements are fundamentally based on both EPRI TR-103412 and EPRI TR-102371 covering a variety of information for the performance assessment of plant instrumentation under severe accident conditions. As an alternative device to real systems, the simulator makes it possible for operators to take a correct action through a reasonable assessment of the performance of the plant instrumentation, even for severe accidents. The ASSA module system has been designed through a realization of a one body order system of one program linked to signal analysis tool programs and to an abnormal signal simulation engine as a one order command. As a result, the program will be able to analyze data quickly and conveniently, onsite.

Using the ASSA module system, we could obtain the response characteristics from the output voltage levels of a pulse parameter according to a change of the resistance for each of $R_{2}$, and $R_{3}$, and the response characteristic from the output voltage level data and time constant of the pulse parameter according to a change of the capacitance for each of $C_{1}$, and $C_{2}$. For the case of the pulse parameter following a change of the resistance, we obtained good response characteristics for the output voltage level data. For the case of the pulse parameter following a change of the capacitance, we could obtain much better response characteristics for the output voltage level data including the time constant patterns. With the ASSA module system, we additionally obtained a type of the varying signal patterns by a simple change of another element's $R-C$ values. The forgoing function of the ASSA module makes it possible to change the abnormal signal patterns by changing the element $R-C$ value and by using specific signal processing. In this paper, we can also obtain a correlative equation between the temperature and the amplitude of an output voltage in the equivalent circuit, which means that an $R_{2}$ has been changing in the resistance range as a distributed parameter when the $T$ temperature has been changing in the temperature range by accident conditions. While the system module makes a signal analysis of noise patterns easy and convenient, it has not been built as a full simulation system as of yet. To make the ASSA module fully available for harsh environments, further research must be focused on developing an $R$ $L-C$ (including active elements: a diode, N-P-N, P-N-P transistors) correlation equation for reflecting the effects of the circumstance parameters such as a high temperature, high pressure, high humidity, and high radiation. Since a variety of simulations to check on malfunctions or abnormal indications from instruments are possible through the ASSA module, it is possible to develop an alternative method to check on a degradation of the instruments with diagnosis logics for severe conditions and a database from the ASSA module analysis results.

\section{Acknowledgment}

This study has been carried out under the Nuclear R\&D Program by the Korean Ministry of Education, Science and Technology.

\section{References}

[1] Fauske \& Associates, "Severe accident management guidance," Tech. Rep. EPRI TR-101869, Fauske \& Associates, Burr Ridge, Ill, USA, 1992.

[2] NUREG/CR-5444, "Instrumentation availability during sever accidents for a boiling water reactor with a MARK 1 containment," Tech. Rep. EGG-2661, 1992.

[3] M. E. Yancey and R. C. Strahm, 1985, Examination and Evaluation of TMI-2 Transmitters CF-1-PT4 and CF-2-LT, GEND-INF-029, Vol. III.

[4] J. Horn, R. W. James, and D. E. True, "Assessment of existing plant instrumentation for severe accident management," Tech. Rep. EPRI TR-103412, 1993.

[5] C. A. Negin et al., "Instrument performance under severe accident conditions," Tech. Rep. EPRI TR-102371, 1993.

[6] K. M. Koo, S. B. Kim, H. D. Kim, and H. Y. Kang, "Design for a new signal analyzer through a circuit modeling simulation as a one body system for SAC's," in Proceedings of the International Congress on Advances in Nuclear Power Plants (ICAPP '06), pp. 1028-1032, Reno, Nev, USA, June 2006.

[7] K. M. Koo, S. B. Kim, H. D. Kim, and H. Y. Kang, "Design for a new signals analyzer through the circuit modeling simulation under severe accident conditions," in Proceedings of the IEEK Fall Conference, vol. 28, pp. 171-174, Seoul, Korea, November 2005.

[8] K. M. Koo, S. B. Kim, H. D. Kim, and H. Y. Kang, "Frequency analysis and new diagnosis by the important circuit modeling simulation including active elements under severe accident conditions," in Proceedings of the Korean Standard Nuclear Power Plant (KSNP '03), pp. 184-186, October 2003.

[9] K. M. Koo, S. B. Kim, H. D. Kim, and H. Y. Kang, "Assessment of existing Ul-Jin $3 \sim 4$ plants instrumentation for severe 
accident management," in Proceedings of the Korean Standard Nuclear Power Plant (KSNP '04), pp. 144-146, June 2004.

[10] B. Jones, 2008, Method and Apparatus for Real-time Pulse Parameter Estimator, Pub. No.: US 2008/0298514 A1. 

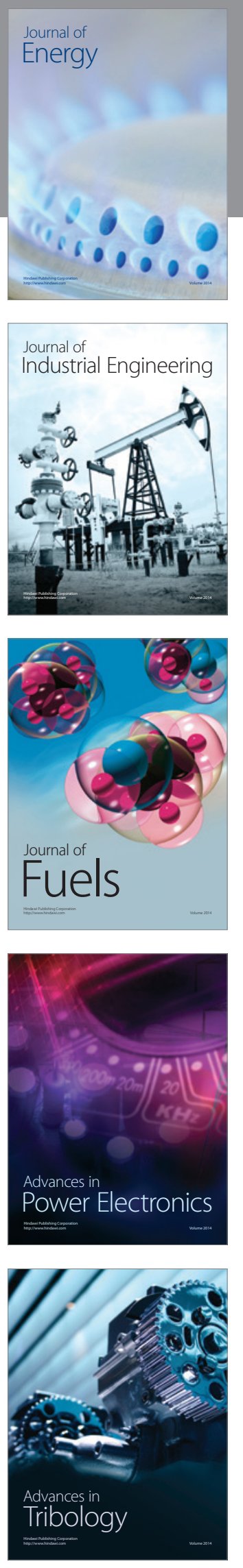
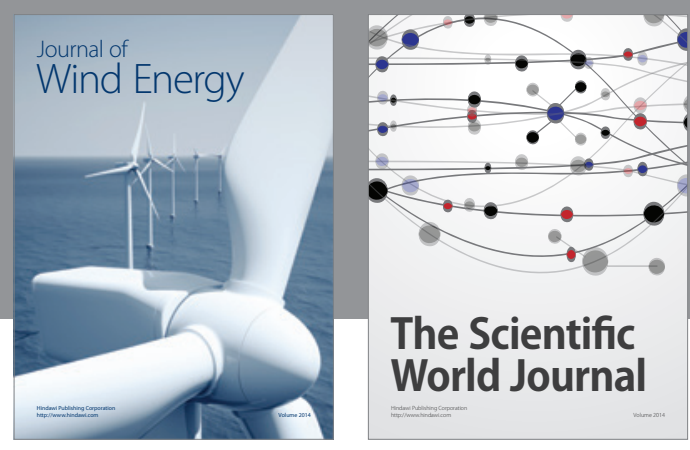

The Scientific World Journal

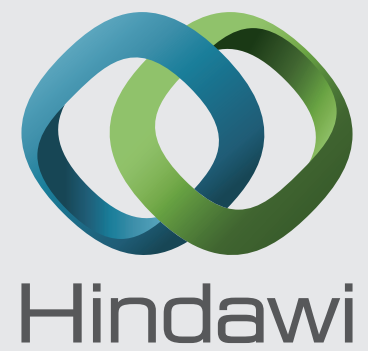

Submit your manuscripts at http://www.hindawi.com
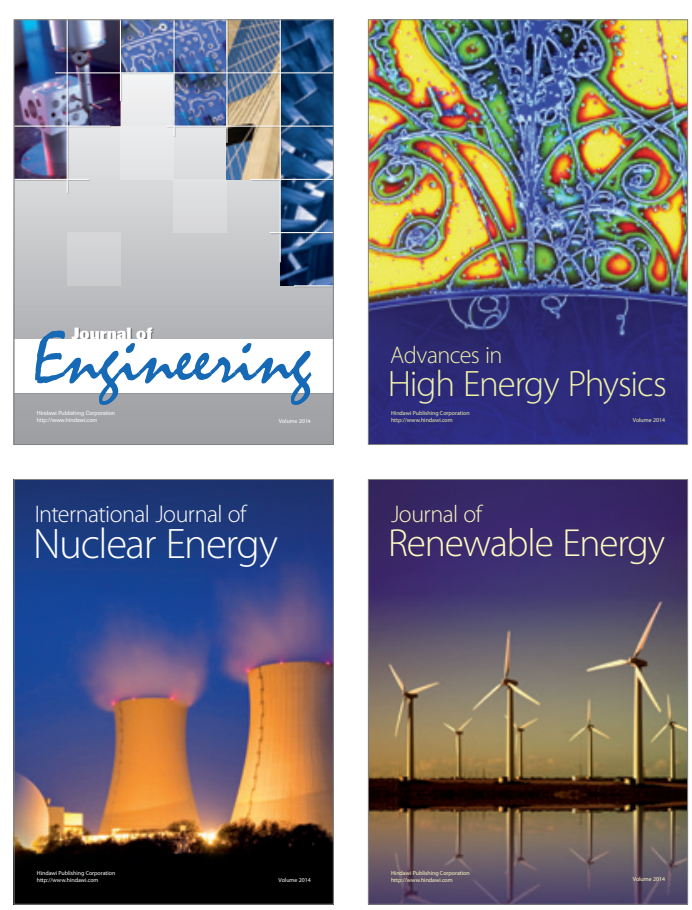

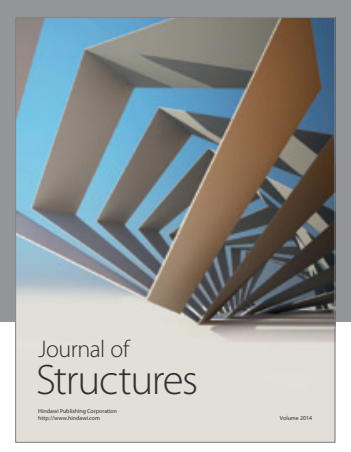

Rotating
Mechinery
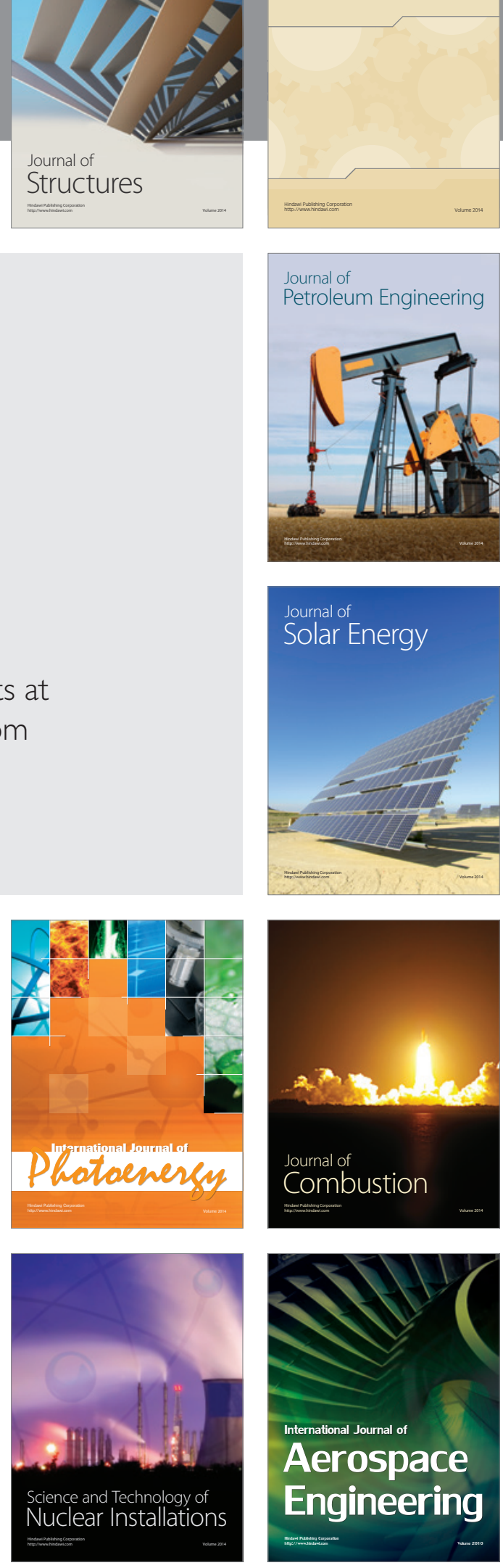